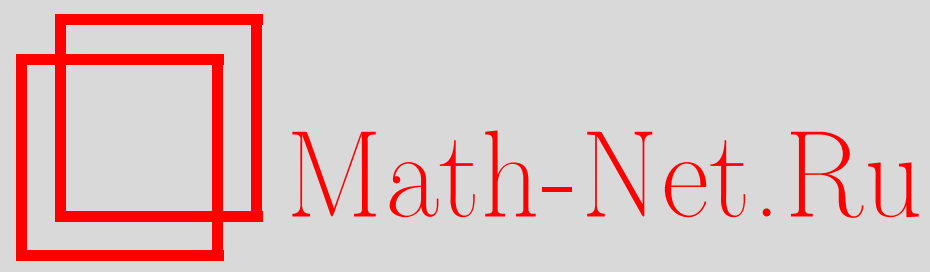

В. В. Грушин, Асимптотическое поведение собственных значений оператора Шрёдингера с поперечным потенциалом в слабо искривленных бесконечных цилиндрах, $M a-$ тем. заметки, 2005, том 77, выпуск 5, 656-664

DOI: https://doi.org/10.4213/mzm2524

Использование Общероссийского математического портала Math-Net.Ru подразумевает, что вы прочитали и согласны с пользовательским соглашением http://www . mathnet.ru/rus/agreement

Параметры загрузки:

IP : 35.173 .219 .12

26 апреля 2023 г., $16: 10: 45$

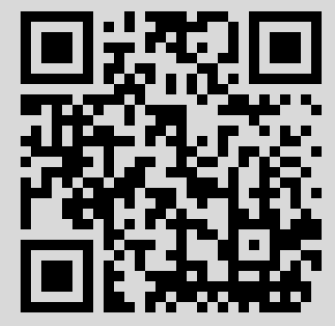




\section{АСИМПТОТИЧЕСКОЕ ПОВЕДЕНИЕ СОБСТВЕННЫХ ЗНАЧЕНИЙ ОПЕРАТОРА ШРЁДИНГЕРА С ПОПЕРЕЧНЫМ ПОТЕНЦИАЛОМ В СЛАБО ИСКРИВЛЕННЫХ БЕСКОНЕЧНЫХ ЦИЛИНДРАХ}

В.В. Грушин

В работе приведены достаточные условия существования собственного значения для оператора Лапласа и оператора Шрёдингера с поперечным потенциалом с нулевьпи условиями Дирихле в трубках, т.е. искривленных и скрученных бесконечных цилиндрах. Для трубок с малой кривизной и малым внутренним кручением получена асимтотическая формула для собственного значения. Показано, что при определенных соотношениях между величинами кривизны и внутреннего кручения трубки дискретньй спектр таких операторов может отсутствовать.

Библиография: 21 название.

1. Введение. В статье изучается поведение собственных значений оператора Лапласа в трубках - трехмерных бесконечных изогнутых и скрученных цилиндрах (точное определение будет дано в п. 2) и в искривленных плоских полосках. Рассматривается также оператор Шрёдингера $H$ с потенциалом $V$, зависящим от поперечных координат. Наряду с чисто математическим интересом к вопросам такого рода, им уделяется в последнее время большое внимание в физической литературе в связи с развитием так назьваемых "нанотехнологий", позволяющих создавать реальные объекты, похожие на трубки (они назьваются квантовыми проводниками, а собственные функции связанньми состояниями). Нужно отметить, что ранее подобные вопросы рассматривались в связи с теорией волноводов и резонаторов. В работах В. П. Маслова [1], [2], В.П. Маслова и Е. М. Воробьёва [3] была построена асимптотика собственных функций и квазистационарных собственных значений уравнения $\Delta u+k^{2} u=0$ с краевыми условиями на эквидистантных кривых. Было показано, что, подбирая нужным образом искривленность полоски и длину участка, где происходит искривление, можно добиться, чтобы существовало лишь одно собственное значение, т.е. резонатор имел лишш одну поперечную моду. Аналогичные вопросы исследовались для областей, ограниченных эквидистантными поверхностями.

Изучению связанных состояний в трубках и приложениям этих вопросов к задачам квантовой механики посвящено большое количество работ. В работах В.В. Белова, С. Ю. Доброхотова, С. О. Синицьна [4], В. В. Белова, С. Ю. Доброхотова, С. О. Синицына, Т. Я. Тудоровского [5], В. В. Белова, С. Ю. Доброхотова, Т. Я. Тудоровского [6], [7] с помощью канонического оператора В.П. Маслова построены быстроосциллирующие решения уравнения Шрёдингера и асимптотические собственные значения в тонких 
трубках. Изучению связанных состояний в трубках посвящены работы П. Дюкло и П. Экснера [8], П. Экснера [9]-[11], В. Булла, Ф. Гезтези, Б. Саймона [12], М. Энтина и М. Магарилла [13], [14].

Основной результат данной статьи сформулирован в п. 2 в виде теоремы 1 , в которой приводится асимптотическая формула (9) для собственных значений оператора Шрёдингера $H$ с потенциалом $V$, зависящим от поперечньг координат, в трубках с малыми кривизной и внутренним кручением, причем наличие или отсутствие собственных значений (удовлетворяющих условию (8)) определяется знаком числа $\mu_{2}$, вычисленного по формуле (6). Доказательство этой теоремы приведено в п. 5. Оно использует некоторую модификацию результатов статьи [15], приведенную в п. 3 и формулу (13) из п. 4 , выражающую оператор Лапласа в криволинейных координатах (2), естественным образом связанньх с трубкой. В п. 6 вычисляется существенньй спектр оператора $H$.

На важность рассмотрения потенциала, зависящего от поперечных координат, поскольку в приложениях такой потенциал отвечает взаимодействию с кристаллом (см. [6], [7]), автору указал В.В. Белов. Отметим, что теорема 2 в принципе позволяет получать асимптотики типа (9) и при наличии некоторых других дополнительных к $V$ потенциалов (с финитными носителями, имеющими первый порядок малости относительно параметра $\varepsilon$, характеризуюшего малость кривизны и внутреннего кручения трубки см. п. 2).

2. Формулировка основного результата. Пусть в $\mathbb{R}^{3}$ имеется гладкая несамопересекающаяся кривая $\Gamma$, заданная уравнением $x=X(s)$, где $s$ - длина дуги (со знаком) кривой $\Gamma$, отсчитанная от фиксированной точки $x_{0} \in \Gamma$. Будем предполагать, что кривая $Г$ снабжена трехгранником $Ф$ рене, состоящим из взаимно ортогональных единичных векторов $(\mathbf{v}(s), \mathbf{n}(s), \mathbf{b}(s))$ класса $C^{\infty}, \mathbf{v}(s)=\dot{X}(s)$, удовлетворяющих системе уравнений $\Phi$ рене $\dot{\mathbf{v}}=\gamma \mathbf{n}, \dot{\mathbf{n}}=-\gamma \mathbf{v}+\varkappa \mathbf{b}, \dot{\mathbf{b}}=-\varkappa \mathbf{v}$, где $\gamma(s)-$ кривизна (со знаком), $\varkappa(s)-$ кручение кривой $\Gamma$, точка здесь и далее означает производную по $s$.

Пусть на плоскости $\mathbb{R}^{2}$ с координатами $\left(q_{1}, q_{2}\right)$ имеется связная ограниченная область $M$ с бесконечно гладкой границей $\partial M$. Обозначим через $\Pi(s)$ нормальную к кривой Г плоскость, в которой векторы $\mathbf{n}(s)$ и $\mathbf{b}(s)$ образуют базис. Рассмотрим в П $(s)$ базис $\left(\mathbf{n}_{\phi}(s), \mathbf{b}_{\phi}(s)\right)$, образованный поворотом базиса $(\mathbf{n}(s), \mathbf{b}(s))$ на угол $\phi(s) \in C^{\infty}(\mathbb{R})$. Трубкой будем назьвать область $\Omega$ в $\mathbb{R}^{3}$, которая получается как образ прямой трубки (цилиндра)

$$
\widetilde{\Omega}=\{(s, q):-\infty<s<+\infty, q \in M\}, \quad q=\left(q_{1}, q_{2}\right),
$$

при отображении

$$
x=X(s)+q_{1} \mathbf{n}_{\phi}(s)+q_{2} \mathbf{b}_{\phi}(s)=X(s)+\left(q_{1} \cos \phi-q_{2} \sin \phi\right) \mathbf{n}+\left(q_{1} \sin \phi+q_{2} \cos \phi\right) \mathbf{b} .
$$

Будем предполагать, что отображение (1) в замыкании $\widetilde{\Omega}$ есть диффеоморфизм. Таким образом, $\left(s, q_{1}, q_{2}\right)$ представляют собой криволинейные координаты в $\bar{\Omega}$. Трубку можно наглядно представлять себе как результат скручивания и изгибания бесконечного цилиндра, либо область, которую заметает плоская фигура $M$ при таком движении ее плоскости, что одна из точек этой плоскости движется вдоль кривой $Г$, а сама эта плоскость остается нормальной к кривой Г. В дальнейшем будем предполагать, что $\gamma \in C_{0}^{\infty}(\mathbb{R})$ и носитель $\gamma$ расположен в отрезке $[0, l]$, т.е. Гпри $s<0$ и $s>l$ превращается в прямые линии. 
Сформулируем основной результат статьи. Ведем углы $\alpha(s)$ и $\theta(s)$, удовлетворяющие соотношениям

$$
\dot{\alpha}(s)+\varkappa(s)=0, \quad \alpha(s)+\theta(s)=\phi(s) .
$$

Угол $\theta(s)$, следуя работе [5], будем называть внутренним кручением трубки. Будем рассматривать трубки с малыми $\gamma(s)$ и $\dot{\theta}(s)$, заменив $\gamma(s)$ и $\dot{\theta}(s)$ на $\varepsilon \gamma(s)$ и $\varepsilon \dot{\theta}(s)$, где $\varepsilon-$ мальй параметр; функции $\gamma, \alpha$ и $\theta$ считаются фиксированными, причем $\gamma(s)$ и $\dot{\theta}(s)$ принадлежат $C_{0}^{\infty}(\mathbb{R})$.

Известно, что для определения кривой с точностью до движения в евклидовом пространстве можно произвольным образом задавать ее кривизну и кручение, поэтому $\Omega$ теперь будем рассматривать как трубку, построенную по этим новым характеристикам (ясно, что при малых $\varepsilon$ условие диффеоморфности отображения (2) будет вьполнено).

В трубке $\Omega$ рассмотрим оператор $\Delta_{D}^{\Omega}-$ расширение по $ф$ ридрихсу оператора Лапласа $\Delta$, первоначально определенного на $C_{0}^{\infty}(\Omega)$. Как показано в [15], область определения $\mathscr{D}$ оператора $\Delta_{D}^{\Omega}$ состоит из функций соболевского пространства $\mathscr{H}^{2}(\Omega)$, удовлетворяющих нулевому условию Дирихле (т.е. обращающихся в нуль на гранище области $\Omega$ ). Напомним, что $u \in \mathscr{H}^{2}(\Omega)$, если функция $u$ и ее обобщенные производные до второго порядка включительно принадлежат $L_{2}(\Omega)$.

Мы будем искать собственные значения и собственные функции оператора

$$
H=-\Delta_{D}^{\Omega}+V
$$

т.е. решения $u \in \mathscr{D}, u \not \equiv 0$ уравнения

$$
\Delta u-V u+\lambda u=0 .
$$

Относительно “потенциала" $V$ будем предполагать, что он вещественньй, $V \in C$ ( $(\bar{\Omega})$ и зависит только от поперечных координат, т.е. в координатах $\left(s, q_{1}, q_{2}\right)$ он не зависит от $s$. В частном случае $V=0$ мы получаем собственные функции оператора Лапласа.

Так как $H$ самосопряжен, его собственные значения (если они существуют) вещественны. Используя теоремы о локальной гладкости решений эллиптических краевых задач (см., например, [16]) и методы статьи [17], можно доказать, что собственные функции оператора $H$ бесконечно дифференцируемы в $\bar{\Omega}$ и вместе со всеми производными экспоненциально убывают при $x$, стремящемся к бесконечности (мы не будем использовать этот факт в данной статье).

Пусть $\left\{\chi_{n}\right\}$ - полная ортонормированная система собственных функций оператора $-\partial_{q_{1}}^{2}-\partial_{q_{2}}^{2}+V\left(q_{1}, q_{2}\right)$ в области $M$ с нулевым условием Дирихле,

$$
\nu_{0}<\nu_{1} \leqslant \nu_{2} \leqslant \cdots \leqslant \nu_{n} \leqslant \cdots
$$

- соответствующие собственные значения. Функции $\chi_{n}$ можно выбрать вещественными. Хорошо известно, что основное собственное значение $\nu_{0}$ невырожденно и $\chi_{0}$ можно выбрать положительной в $M$ (см., например, [18]).

Введем следующие обозначения:

$$
\begin{gathered}
\beta=\gamma\left(q_{1} \cos \phi-q_{2} \sin \phi\right), \quad L=q_{1} \partial_{q_{2}}-q_{2} \partial_{q_{1}}, \\
\rho_{n}=\sqrt{\nu_{n}-\nu_{0}}, \quad \mathscr{E}_{\rho_{n}}=-\frac{1}{2 \rho_{n}} e^{-\rho_{n}|s|},
\end{gathered}
$$


и определим число $\mu_{2}$ формулой

$$
\begin{aligned}
\mu_{2}= & -\frac{1}{8} \sum_{n=1}^{\infty} \rho_{n}^{4} \int_{\mathbb{R}} \beta_{n 0} \mathscr{E}_{\rho_{n}} * \beta_{n 0} d s+\frac{1}{2} \sum_{n=1}^{\infty} \rho_{n}^{2}\left(L_{0 n}\right)^{2} \int_{\mathbb{R}} \dot{\theta} \mathscr{E}_{\rho_{n}} * \dot{\theta} d s \\
& -\frac{1}{2} \sum_{n=1}^{\infty} \rho_{n}^{2} L_{n 0} \int_{\mathbb{R}} \dot{\beta}_{n 0} \mathscr{E}_{\rho_{n}} * \dot{\theta} d s .
\end{aligned}
$$

Здесь используется стандартное в квантовой механике обозначение

$$
A_{m n}=\int_{M} \bar{\chi}_{m} A \chi_{n} d q
$$

знак "звездочка" означает свертку функций.

ТЕорема 1. Если $\mu_{2}>0$, то при некоторых $\varepsilon_{0}>0 u \mu_{0}>0$ для любого $0<\varepsilon<\varepsilon_{0}$ существует единственное собственное значение оператора $H$, удовлетворяющее неравенству

$$
\lambda<\nu_{0}+\mu_{0}^{2}
$$

Это собственное значение невырожденно и имеет при $\varepsilon \rightarrow 0$ асимптотику

$$
\lambda=\nu_{0}-\mu^{2}, \quad \mu=\varepsilon^{2} \mu_{2}+O\left(\varepsilon^{3}\right) .
$$

Если же $\mu_{2}<0$, то собственных значений, удовлетворяющих неравенству (8) для $0<\varepsilon<\varepsilon_{0}$ не существует.

Доказательство этой теоремы использует некоторую модификацию результатов статьи [15], приведенную в п. 3, и будет дано в п. 5. В частном случае, когда $Г$ - плоская кривая и $\phi=0$, и в случае, когда $\Gamma$ - прямая линия (т.е. $\gamma=0$ ), теорема 1 была получена в [15]. В случае $\theta=0$ при несколько более общих предположениях на кривизну кривой $\Gamma$ формула, по существу эквивалентная (9), была получена другим методом в [8].

Заметим, что при $\gamma \not \equiv 0$ первое слагаемое в (6) положительно. Действительно, для любой вещественной функции $f(s) \in C_{0}^{\infty}(\mathbb{R})$ и $f(s) \not \equiv 0$ интеграл от $f(s) \mathscr{E}_{\rho_{n}} * f(s)$ по вещественной оси положителен, что следует из равенства Парсеваля, если перейти к преобразованию Фурье. Поэтому первое слагаемое в (6) неотрицательно. Оно может равняться нулю, только если $\beta_{n 0}(s) \equiv 0$ при всех $n \geqslant 1$, откуда следует, что $\beta(s)=C(s) \chi_{0}(q)$, но в таком случае получается, что $\beta(s)=0$ на $\partial M$; следовательно, $\gamma(s) \equiv 0$. Если $\gamma \not \equiv 0$, то это приводит к тому, что $\mu_{2}>0$ при $\dot{\theta}(s) \equiv 0$ или достаточно малых $\dot{\theta}(s)$; следовательно, $H$ в таких случаях при достаточно малых $\varepsilon$ имеет хотя бы одно собственное значение. В п. 6 будет доказано, что множество $\lambda \geqslant \nu_{0}$ является существенным спектром оператора $H$ (определение дискретного и существенного спектра см. в [19]); следовательно, это собственное значение принадлежит дискретному спектру. Отметим, что для трубок без внутреннего кручения (т.е. при $\theta(s) \equiv 0)$ с произвольной кривизной $\gamma(s) \in C_{0}^{\infty}(\mathbb{R})$ (не обязательно малой) существование дискретного спектра было доказано в [8].

Аналогичным образом доказьвается, что второе слагаемое в (6) неположительно и равно нулю, только если $\dot{\theta}(s) \equiv 0$ или $\dot{\theta}(s) \not \equiv 0$, но область $M$ является кольцом, граница 
которого состоит из двух концентрических окружностей с центрами в начале координат (подробно это рассуждение приведено в [15]). Если $M$ таким кольцом не является и $\dot{\theta}(s) \not \equiv 0$, то второе слагаемое в (6) отрицательно; в таком случае $\mu_{2}<0$ при $\gamma(s) \equiv 0$ или при достаточно малых $\gamma(s)$; следовательно, $H$ при достаточно малых $\varepsilon$ не имеет собственных значений, удовлетворяющих неравенству (8), и дискретный спектр у $H$ отсутствует.

3. Возмущенный оператор Лапласа в бесконечном цилиндре. Пусть $\widetilde{\Omega}-$ бесконечный цилиндр, определенный в (1). Рассмотрим дифференциальньй оператор

$$
P_{\mu, \varepsilon}=-\left(\partial_{s}^{2}+\partial_{q_{1}}^{2}+\partial_{q_{2}}^{2}\right)+V+\varepsilon A+\varepsilon^{2} B+T-\left(\nu_{0}-\mu^{2}\right),
$$

где $A\left(s, q, \partial_{s}, \partial_{q_{1}}, \partial_{q_{2}}\right)$ и $B\left(s, q, \partial_{s}, \partial_{q_{1}}, \partial_{q_{2}}\right)$ - линейные дифференциальные операторы второго порядка с коэффициентами из $C^{\infty}$ в замькании $\widetilde{\Omega}$, коэффициенты $T\left(\mu, \varepsilon, s, q, \partial_{s}\right.$, $\left.\partial_{q_{1}}, \partial_{q_{2}}\right)$ определены, когда $(\mu, \varepsilon)$ изменяются в окрестности точки $(0,0)$, a $(s, q)$ - в замыкании $\widetilde{\Omega}$, бесконечно дифференцируемы по совокупности переменных и аналитичны по $(\mu, \varepsilon)$. Функция $V$ вещественна, зависит только от $\left(q_{1}, q_{2}\right)$ и принадлежит $C^{\infty}(\bar{M})$. Будем предполагать, что коэффициенты $A, B$ и $T$ финитны и их носители расположены в $[0, l] \times \bar{M}$ при некотором $l>0$. Кроме того, будем предполагать, что коэффициенты оператора $T$ при $(\mu, \varepsilon)=(0,0)$ обращаются в нуль вместе с производными по переменным $(\mu, \varepsilon)$ до второго порядка включительно, что символически можно записать как $T=O\left(|\varepsilon|^{3}+|\mu|^{3}\right)$. Будем рассматривать $P_{\mu, \varepsilon}$ как непрерьвньй оператор из $\mathscr{H}_{D}^{2}(\widetilde{\Omega})$ в $L_{2}(\widetilde{\Omega})$, где $\mathscr{H}_{D}^{2}(\widetilde{\Omega})$ - подпространство функций соболевского пространства $\mathscr{H}^{2}(\widetilde{\Omega})$, обращающихся в нуль на границе области $\widetilde{\Omega}$. Через $\operatorname{Ker} P_{\mu, \varepsilon}$, как обычно, обозначается подпространство решений уравнения $P_{\mu, \varepsilon} u=0$.

Зададим числа $\mu_{1}$ и $\mu_{2}$ формулами

$$
\begin{aligned}
\mu_{1}= & -\frac{1}{2} \int_{\mathbb{R}} A_{00}(s) d s \\
\mu_{2}= & -\frac{1}{2} \int_{\widetilde{\Omega}} \bar{\chi}_{0}(q)\left(\mu_{1}[A, s]+\int_{0}^{s}\left(s-s^{\prime}\right) A_{00}\left(s^{\prime}\right) d s\right) \chi_{0}(q) d s d q \\
& -\frac{1}{2} \int_{\mathbb{R}} B_{00}(s) d s-\frac{1}{2} \sum_{n=1}^{\infty} \int_{\widetilde{\Omega}} \bar{\chi}_{0}(q) A\left(\left(\mathscr{E}_{\rho_{n}} * A_{n 0}\right) \chi_{n}(q)\right) d s d q,
\end{aligned}
$$

где $A_{m n}$ определяются формулой $(7),[A, s]$ - коммутатор $A$ и оператора умножения на $s$, $\mathscr{E}_{\rho_{n}}$ определены в (5). Нам понадобится следующая модификация теоремы 1.1 из статьи [15], доказательство которой получается почти дословным повторением соответствующего доказательства из [15].

Теорема 2. Если $\operatorname{Re} \mu_{1}>0$ или $\operatorname{Re} \mu_{1}=0 u \operatorname{Re} \mu_{2}>0$, mo при некоторых $\varepsilon_{0}>0$ и $\mu_{0}>0$ для любого $0<\varepsilon<\varepsilon_{0}$ существует единственное значение $\mu$, удовлетворяющее неравенству $|\mu|<\mu_{0}$, при котором $\operatorname{Ker} P_{\mu, \varepsilon} \neq 0$. При таком $\mu \operatorname{dim} \operatorname{Ker} P_{\mu, \varepsilon}=1$. Указанное значение $\mu$ имеет при $\varepsilon \rightarrow 0$ асимптотику

$$
\mu=\varepsilon \mu_{1}+\varepsilon^{2} \mu_{2}+O\left(\varepsilon^{3}\right) .
$$

$B$ случае вещественных коэффициентов операторов $A, B$ и $T$ такое значение $\mu$ вешественно.

Если же $\operatorname{Re} \mu_{1}<0$ или $\operatorname{Re} \mu_{1}=0 u \operatorname{Re} \mu_{2}<0$, mо $\operatorname{Ker} P_{\mu, \varepsilon}=0$ при некоторых $\varepsilon_{0}>0 u \mu_{0}>0$ для любых $0<\varepsilon<\varepsilon_{0} u|\mu|<\mu_{0}$. 
4. Оператор Лапласа в координатах, связанных с трубкой. В этом пункте мы получим выражение для оператора Лапласа в координатах (2). Введем углы $\alpha(s)$ и $\theta(s)$, удовлетворяющие соотношениям $(3)$, и пусть

$$
g(s)=1-\gamma(s)\left(q_{1} \cos \phi(s)-q_{2} \sin \phi(s)\right), \quad L=\left(q_{1} \partial_{q_{2}}-q_{2} \partial_{q_{1}}\right) .
$$

В координатах $\left(s, q_{1}, q_{2}\right)$ оператор Лапласа приобретает вид

$$
g^{-1}\left(\partial_{s}-\dot{\theta} L\right) g^{-1}\left(\partial_{s}-\dot{\theta} L\right)+g^{-1} \partial_{q_{1}} g \partial_{q_{1}}+g^{-1} \partial_{q_{2}} g \partial_{q_{2}}
$$

Эту формулу можно получить, непосредственно используя известное выражение оператора Лапласа в криволинейной системе координат через метрический тензор (cp. c [4]-[7]). Но этот путь приводит к довольно громоздким вычислениям, поскольку система координат $\left(s, q_{1}, q_{2}\right)$ в общем случае не триортогональная. Здесь мы используем для вывода формулы (13) специальную промежуточную триортогональную систему координат $\left(s, \tau_{1}, \tau_{2}\right)$ (такие координаты использовались в [5]-[8], [14]).

Пусть $\left(\tau_{1}, \tau_{2}\right)$ - координаты в плоскости П $(s)$ в базисе $\left(\mathbf{n}_{\alpha}(s), \mathbf{b}_{\alpha}(s)\right)$, полученном из $(\mathbf{n}(s), \mathbf{b}(s))$ поворотом на угол $\alpha(s)$. Система координат $\left(s, \tau_{1}, \tau_{2}\right)$ является триорто-

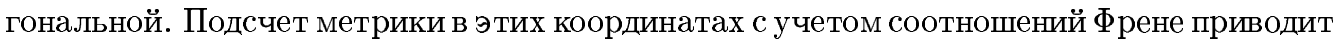
к простому выражению

$$
d x^{2}=g^{2} d s^{2}+d \tau_{1}^{2}+d \tau_{2}^{2}, \quad g=1-\gamma\left(\tau_{1} \cos \alpha-\tau_{2} \sin \alpha\right)
$$

Поэтому оператор Лапласа в координатах $\left(s, \tau_{1}, \tau_{2}\right)$ имеет вид

$$
g^{-1} \partial_{s} g^{-1} \partial_{s}+g^{-1} \partial_{\tau_{1}} g \partial_{\tau_{1}}+g^{-1} \partial_{\tau_{2}} g \partial_{\tau_{2}}
$$

причем $g$ является якобианом при переходе к координатам $\left(s, \tau_{1}, \tau_{2}\right)$. После поворота базиса $\left(\mathbf{n}_{\alpha}(s), \mathbf{b}_{\alpha}(s)\right)$ на угол $\theta(s)$ получаем базис $\left(\mathbf{n}_{\phi}(s), \mathbf{b}_{\phi}(s)\right)$, и остается только пересчитать $(14)$ из координат $\left(s, \tau_{1}, \tau_{2}\right)$ в координаты $\left(s, q_{1}, q_{2}\right)$, используя простые соотношения $\tau_{1}=q_{1} \cos \theta-q_{2} \sin \theta, \tau_{2}=q_{1} \sin \theta+q_{2} \cos \theta$. В результатеполучим формулу (13), причем $g$ будет якобианом при переходе от координат $x$ к $\left(s, q_{1}, q_{2}\right)$.

5. Доказательство теоремы 1. Заменим в формулах (12) и (13) $\gamma(s)$ и $\dot{\theta}(s)$ на $\varepsilon \gamma(s)$ и $\varepsilon \dot{\theta}(s)$; соответствующим образом $g$ теперь будет определяться формулой

$$
g=1-\varepsilon \beta, \quad \beta=\gamma\left(q_{1} \cos \phi-q_{2} \sin \phi\right) .
$$

Положим $\lambda=\nu_{0}-\mu^{2}$. В силу формулы (13) после умножения на $g$ уравнение (4) для собственных функций оператора $H$ в координатах $\left(s, q_{1}, q_{2}\right)$ представляется в виде

$$
-\left(\partial_{s}^{2}+\partial_{q_{1}}^{2}+\partial_{q_{2}}^{2}\right) u+V u+\varepsilon A u+\varepsilon^{2} B u+T u-\left(\nu_{0}-\mu^{2}\right) u=0,
$$

где

$$
\begin{aligned}
& A=\partial_{s} \dot{\theta} L+\dot{\theta} L \partial_{s}-\partial_{s} \beta \partial_{s}+\partial_{q_{1}} \beta \partial_{q_{1}}+\partial_{q_{2}} \beta \partial_{q_{2}}-\beta V+\nu_{0} \beta \\
& B=-\dot{\theta}^{2} L^{2}-\partial_{s} \beta^{2} \partial_{s}+\partial_{s} \beta \dot{\theta} L+\dot{\theta} L \beta \partial_{s} .
\end{aligned}
$$


К этому уравнению мы применим теорему 2. Учитывая соотношение

$$
\begin{aligned}
\int_{M} \bar{\chi}_{n}\left(\left(\partial_{q_{1}} \beta\right)\left(\partial_{q_{1}} \chi_{0}\right)+\left(\partial_{q_{2}} \beta\right)\left(\partial_{q_{2}} \chi_{0}\right)\right) d q & =\frac{1}{2} \int_{M} \bar{\chi}_{n}\left(\partial_{q_{1}}^{2}+\partial_{q_{2}}^{2}-V+\nu_{0}\right)\left(\beta \chi_{0}\right) d q \\
& =-\rho_{n}^{2} \beta_{n 0},
\end{aligned}
$$

получаем, что $A_{00}=0$, поэтому $\mu_{1}=0$, где $\mu_{1}$ определяется формулой $(10)$. Теперь формула (11) для $\mu_{2}$ запишется в виде

$$
\mu_{2}=\frac{1}{2} \int_{\mathbb{R}} \dot{\theta}^{2}\left(L^{2}\right)_{00} d s-\frac{1}{2} \sum_{n=1}^{\infty} \int_{\mathbb{R}}\left(\dot{\theta} L_{0 n} \partial_{s}-\frac{1}{2} \rho_{n}^{2} \beta_{0 n}\right) \mathscr{E}_{\rho_{n}} *\left(\ddot{\theta} L_{n 0}-\frac{1}{2} \rho_{n}^{2} \beta_{n 0}\right) d s .
$$

Формула (6) получается из (15) после преобразований, использующих интегрирования по частям и соотношения $\beta_{0 n}=\beta_{n 0}, L_{0 n}=-L_{n 0}$. Например, интегрируя по частям и используя соотношение $\ddot{\mathscr{E}}_{\rho_{n}}(s)=\rho_{n}^{2} \mathscr{E}_{\rho_{n}}(s)+\delta(s)$, получим, что

$$
\int_{\mathbb{R}} \dot{\theta} \partial_{s} \mathscr{E}_{\rho_{n}} * \ddot{\theta} d s=\int_{\mathbb{R}} \dot{\theta}^{2} d s+\rho_{n}^{2} \int_{\mathbb{R}} \dot{\theta} \partial_{s} \mathscr{E}_{\rho_{n}} * \theta d s .
$$

В силу равенства Парсеваля

$$
\sum_{n=1}^{\infty} L_{0 n} L_{n 0}=-\sum_{n=1}^{\infty} L_{n 0}^{2}=-\int_{M}\left(L \chi_{0}\right)^{2} d q=\left(L^{2}\right)_{00}
$$

Подставляя эти выражения в (15) (преобразование остальных интегралов не вызьвает затруднений), мы и получаем окончательную формулу (6). В результате утверждение теоремы 1 с заменой неравенства (8) на неравенство $\left|\lambda-\nu_{0}\right|<\mu_{0}^{2}$ будет доказано. Остается заметить, что при достаточно малом $\varepsilon_{0}$ для $0<\varepsilon<\varepsilon_{0}$ оператор $H$ не имеет собственных значений с $\lambda<\nu_{0}-\mu_{0}^{2}$. Для этого достаточно показать, что

$$
\left(\left(H-\nu_{0}\right) u, u\right) \geqslant-\mu_{0}^{2}(u, u)
$$

для любых $u \in \mathscr{H}_{D}^{2}(\Omega)$, где $(u, v)$ - скалярное произведение в $L_{2}(\Omega)$. Перейдем к координатам $\left(s, q_{1}, q_{2}\right)$ и положим

$$
H_{0}=-\left(\partial_{s}^{2}+\partial_{q_{1}}^{2}+\partial_{q_{2}}^{2}\right)+V .
$$

$\mathrm{C}$ помощью разложения по собственным функциям $\chi_{n}$ легко устанавливается, что оператор $H_{0}-\nu_{0}$ неотрицательно определен относительно скалярного произведения в $L_{2}(\widetilde{\Omega})$. Как видно из формулы $(13), g H-H_{0}$ есть дифференциальньй оператор, коэффищиенты которого имеют порядок $O(\varepsilon)$. С помощью интегрирования по частям и неравенства Коши-Буняковского теперь легко получаются следующие оценки при достаточно малых $\varepsilon$ :

$$
\begin{aligned}
\left(\left(H-\nu_{0}\right) u, u\right) & =\int_{\widetilde{\Omega}} \bar{u} g\left(H-\nu_{0}\right) u d s d q \\
& \geqslant \int_{\widetilde{\Omega}} \bar{u}\left(H-\nu_{0}\right) u d s d q-C \varepsilon \int_{\widetilde{\Omega}} \bar{u}\left(H-\nu_{0}+1\right) u d s d q \\
& \geqslant-C \varepsilon \int_{\widetilde{\Omega}}|u|^{2} d s d q \geqslant-C \varepsilon\left(1+C_{1} \varepsilon\right) \int_{\widetilde{\Omega}} g|u|^{2} d s d q \\
& =-C \varepsilon\left(1+C_{1} \varepsilon\right)(u, u),
\end{aligned}
$$

где $C$ и $C_{1}$ - некоторые положительные константы. Зафиксировав $\mu_{0}$ и выбрав $\varepsilon_{0}$ достаточно малым, получим неравенство (16). Тем самым, теорема 1 доказана. 
6. Существенный спектр оператора $H$. В этом пункте мы докажем следуюшее утверждение.

Tеорема 3. Существенный спектр $\sigma_{\mathrm{ess}}(H)$ оператора $H$ совпадает $c\left[\nu_{0},+\infty\right)$.

ДокАЗАТЕЛЬСтво. Возьмем любое $\lambda \geqslant \nu_{0}$ и покажем, что $\lambda \in \sigma_{\mathrm{ess}}(H)$. Для этого возьмем такую функцию $f(s) \in C_{0}^{\infty}(\mathbb{R})$, что

$$
\int_{\mathbb{R}}|f(s)|^{2} d s=1,
$$

и рассмотрим последовательность функций

$$
u_{n}=\frac{1}{\sqrt{n}} \exp \left(i s \sqrt{\lambda-\nu_{0}}\right) f\left(\frac{s}{n}-n^{2}\right) \chi_{0}(q) \text {. }
$$

Так как при $s>l$ оператор $H$ в координатах $\left(s, q_{1}, q_{2}\right)$ имеет вид $(17)$, то, непосредственно вычисляя выражение $\left(H_{0}-\lambda\right) u_{n}$, получим, что $\left\|(H-\lambda) u_{n}\right\| \rightarrow 0$ при $n \rightarrow \infty$. При достаточно больших $n$ носители функций $u_{n}$ непересекаются между собой и с $[0, l] \times M$; следовательно, соответствующие функции $u_{n}(x)$ при достаточно больших $n$ будут ортогональны, причем $\left\|u_{n}\right\|=1$. Теперь из критерия Вейля (см. [19, теорема VII.11]) следует, что $\lambda \in \sigma_{\text {ess }}(H)$. Тем самым, доказано, что $\left[\nu_{0},+\infty\right) \in \sigma_{\text {ess }}(H)$.

Остается проверить включение $\left(-\infty, \nu_{0}\right) \in \sigma_{\text {disc }}(H)$. Методом статьи [17] легко устанавливается, что при $\operatorname{Re} \lambda<\nu_{0}$ оператор $H-\nu_{0}$ фредгольмов, а поскольку $H$ полуограничен снизу, то для некоторого $m$ он не имеет собственных значений при $\lambda<m$; следовательно, $\operatorname{Ker}(H-\lambda)=0$ и $H-\lambda$ обратим при $\lambda<m$. Из теоремы об аналитических семействах фредгольмовых операторов (см. [20], [21]) следует, что обратньй оператор $R_{\lambda}=(H-\lambda)^{-1}$ при $\operatorname{Re} \lambda<\nu_{0}$ есть мероморфная операторнозначная функция, имеющая в качестве вычетов конечномерные операторы $\left(R_{\lambda}\right.$ рассматривается как оператор из $L_{2}(\Omega)$ в $\left.\mathscr{H}_{D}^{2}(\Omega)\right)$. Это как раз и означает, что при $\operatorname{Re} \lambda<\nu_{0}$ спектр оператора $H$ дискретньй.

\section{СПИСОК ЦИТИРОВАННОЙ ЛИТЕРАТУРЫ}

[1] Маслов В.П. Асимптотика собственньх функций уравнения $\Delta u+k^{2} u=0$ с краевыми условиями на эквидистантных кривых и рассеяние электромагнитных волн в волноводе // Докл. АН СССР. 1958. Т. 123. № 4. С. 631-633.

[2] Maslov V. P. Mathematical Aspects of Integral Optics // Russian J. Math. Phys. 2001. V. 8. P. 83-180.

[3] Маслов В.П., Воробьёв Е. М. Об одномодовых открытых резонаторах // Докл. АН CCCP. 1968. T. 179. №3. C. 558-561.

[4] Белов В. В., Доброхотов С. Ю., Синицын С. О. Асимптотические решения уравнения Шрёдингера в тонких трубках // Тр. ин-та матем. и мех. УрО РАН. 2003. Т. 9. № 1. C. $1-11$.

[5] Белов В. В., Доброхотов С. Ю., Синицын С. О., Тудоровский Т. Я. Квазиклассическое приближение и канонический оператор Маслова для нерелятивистских уравнений квантовой механики в нанотрубках // Докл. РАН. 2003. Т. 393. №4. С. 1-5.

[6] Belov V.V., Dobrokhotov S. Yu., Tudorovskii T. Ya. Quantum and classical dynamics of electron in thin curved tubes with spin and external electromagnetic fields taken into account // Russian J. Math. Phys. 2004. V. 11. № 1. P. 109-118. 
[7] Белов В.В., Доброхотов С. Ю., Тудоровский Т.Я. Асимптотические решения нерелятивистских уравнений квантовой механики в искривленных нанотрубках $1 / /$ TМФ. 2004. T. 141. № 2. C. 267-303.

[8] Duclos P., Exner P. Curvature-induced bound states in quantum waveguides in two and three dimensions // Rev. Math. Phys. 1995. V. 7. P. 73-102.

[9] Exner P. Bound States in curved quantum waveguides // J. Math. Phys. 1989. V. 30. P. 2574-2580.

[10] Exner P. Bound states in quantum waveguides of a slowly decaying curvature // J. Math. Phys. 1993. V. 34. P. 23-28.

[11] Exner P. A quantum pipette // J. Math. Phys. A. 1995. V. 28. P. 5323-5330.

[12] Bulla W., Gesztesy F., Renger W., Simon B. Weakly couped bound states in quantum waveguides // Proc Amer. Math. Soc. 1997. V. 125. P. 1487-1495.

[13] Entin M. V., Magarill L. I. Electrons in twisted quantum wire // Phys. Rev. B. 2002. V. 66. №1-5. P. 205308-1-205308-5.

[14] Магарилл Л.И., Энтин М.В.Электроны в криволинейной квантовой проволоке // ЖЭТФ. 2003. Т. 123. № 4. С. 867-876.

[15] Грушин В.В. О собственньх значениях финитно возмущенного оператора Лапласа в бесконечных цилиндрических областях // Матем. заметки. 2004. Т. 75. № 3. С. 360-371.

[16] Хёрмандер Л. Линейные дифференциальные операторы. М.: Мир, 1965.

[17] Кондратьев В. А. Краевые задачи для эллиптических уравнений в областях с коническими и угловыми точками // Тр. ММО. 1967. Т. 16. С. 207-318.

[18] Рид М., Саймон Б. Методы современной математической физики. Т. 4. Анализ операторов. М.: Мир, 1982.

[19] Рид М., Саймон Б. Методы современной математической физики. Т. 1. Функциональный анализ. М.: Мир, 1982.

[20] Гохберг И. Ц. О линейных операторах, аналитически зависящих от параметра // Докл. AH CCCP. 1951. T. 78. № 4. C. 629-632.

[21] Блехер П. М. Об операторах, мероморфно зависящих от параметра // Вестн. МГУ. Сер. 1. Матем., мех. 1965. № 5. С. 30-36.

Московский государственный институт электроники и математики

Поступило

E-mail: pm@miem.edu.ru

28.04.2004

Исправленный вариант

23.09.2004 